\title{
Article \\ DALI LED Driver Control System for Lighting Operations Based on Raspberry Pi and Kernel Modules
}

\author{
George K. Adam(1) \\ Department of Digital Systems, University of Thessaly, 41110 Larisa, Greece; gadam@uth.gr
}

Received: 26 August 2019; Accepted: 10 September 2019; Published: 12 September 2019

\begin{abstract}
Light emitting diodes (LEDs) as an efficient low-consumption lighting technology are being used increasingly in many applications. The move to LED lighting is also changing the way the lighting control systems are designed. Currently, most electronic ballasts and other digital lighting devices implement the Digital Addressable Lighting Interface (DALI) standard. This paper presents a low-cost, low-power effective DALI LED driver controller, based on open-source Raspberry Pi3 microcontroller prototyping platform. The control software is developed as a Linux kernel module under UBUNTU 18.04.2 LTS patched with PREEMPT_RT (Preemptive Real-time) for real-time processing. This dynamically loaded kernel module performs all the processing, communication and control operations of the Raspberry Pi3-based DALI controller with the DALI LED driver and LED luminaire. Software applications written in C and Python were developed for performance testing purposes. The experimental results showed that the proposed system could efficiently and effectively manage DALI LED drivers and perform lighting operations (e.g., dimming). The system can be used for a variety of purposes from personal lighting control needs and experimental research in control of electronic ballasts and other control gears, devices and sensors, to advanced requirements in professional buildings, including energy management, lighting maintenance and usage.
\end{abstract}

Keywords: Raspberry Pi3; microcontroller; control system; kernel module; DALI protocol

\section{Introduction}

Public lighting installations are important source of energy consumption. Every commercial and residential building consumes a great deal of electrical energy through lighting systems [1-3]. Installations on domestic premises, offices, or other private premises are also considered to be important [4]. In this direction, the work of $\mathrm{Li}$ et al. [5] provides a means to select appropriate photoelectric lighting control systems based on the energy savings. Several control strategies have been proposed for energy efficient office lighting systems design [6,7]. Therefore smart lighting systems should be used for energy management to reduce consumption. Towards this direction, light emitting diodes (LEDs) have proven to be a promising technology since they combine high efficiency, environmental benefits, high reliability and long lifetimes [8]. LEDs are being used increasingly as low-consumption light sources in many applications [9]. The move to LED lighting is also changing the way the lighting control systems are designed [10-12]. LEDs are close to being monochromatic light sources (half-bandwidths of 20-30 nm) and they provide roughly linear light output in response to stimulating control signal. The response time of LED chips is very fast (in the order of nanoseconds) [13].

Lighting control systems use a variety of communication protocols such as DMX (Digital Multiplex Signal), RDM (Remote Device Management), KNX (Konnex Networks) and Digital Addressable Lighting Interface (DALI) to link control devices to lighting device drivers and luminaires [14]. DALI is such a widely-adopted protocol. DALI is a standard communication protocol for lighting control. 
The interface and protocol are defined by the International Electrotechnical Commission as an IEC 60929/EN 60929 standard [15], and were modified by IEC 62386 standard [16]. DALI-2 refers to the latest version of the DALI protocol. For DALI-2 the IEC 62386 standard was reconstructed in late 2014 to include many improvements and additional new commands and features. Most current digital ballasts and other electronic lighting devices (e.g., power switches, sensors and detectors, transformers, etc.), implement the DALI protocol. This is due to its simplicity on wiring and communication needs, based basically on two wires for the transmission of the control signals for commissioning or other maintenance purposes.

Microcontrollers are the heart of any control system, and as such they play a crucial role in commercial and non-commercial lighting systems. Microcontrollers offer the advantages of low-cost, low-power and rapid application development and implementation. The increasing integration of microcontrollers, particularly with PWM (Pulse Width Modulation) control circuits, high resolution timers and other control blocks, allow them to control the lighting systems directly and provide more efficient control at lower costs $[17,18]$. Microcontrollers provide a flexible control platform for handling a wide range of lighting systems found in offices and domestic premises.

Today, microcontroller boards that integrate on a system-on-a-chip (SoC) 32-bit or 64-bit microprocessors have the ability to execute powerful instructions in a single clock cycle, and achieve high throughputs. As such, there is an increasing development, and applications of such systems, towards low-cost, high-performance and digital control of electronic ballasts. Many companies, including Texas Instruments, STMicroelectronics, NXP Semiconductors and Microchip, produce such microcontrollers optimized for lighting control. Such systems, typically integrate analog circuitry combined with digital modules under firmware control $[19,20]$. They also integrate peripherals such as LCD and touchscreen controllers and networking capabilities to high end devices such as smart phones. Today the Internet-of-Things (IoT) revolution has shaped a new application domain where computing in technological applications is typically performed on low-power RISC (Reduced Instruction Set Computer) architectures and general-purpose microprocessors. Raspberry Pi is such an intelligent microcontroller that has been employed in many scientific applications including light control [21-24].

This paper presents the results of research on the implementation of a DALI LED driver control system, based on a Raspberry Pi3 Model B (referred from now on as RPi3) microcontroller board. The choice of RPi3 is based on the fact that it is being extensively used for embedded low-powered control applications, due to its computing power in regard to its price, its ease of use with peripheral equipment and low-energy consumption [25]. In addition, research shows that such a system is capable to support adequate timing for most measurement purposes [26]. The system runs UBUNTU patched with PREEMPT_RT [27] for real-time processing. The main control software is developed as a Linux kernel module. As such, it saves some RAM space since it can be compressed in the same go as the main Linux kernel; that is important in embedded systems with little storage. This module performs all the processing and communication operations of the RPi3 DALI controller with the DALI ballast (DALI LED driver) and LED luminaire, and generates appropriately Manchester encoded control signals for digital lighting operations. It interprets user data and sends to the DALI ballast appropriate DALI commands for commissioning and lighting control operations (e.g., lights on/off and dimming) and other viewing or modification purposes (e.g., maintenance tasks and energy status info).

The novelty of this work is based on the fact that it uses an inexpensive and widely used in many other research and educational purposes ARM (Advanced RISC Machine) development board such as Raspberry Pi, to implement the DALI lighting control protocol architecture, based on open-source dynamic loadable kernel modules, that save RAM storage space, usually limited in embedded devices. Additionally, the control system is enhanced with hard real-time capabilities, based on the PREEMPT_RT real-time patch, installed and configured appropriately, in order to support future advanced timing requirements in control of electronic ballasts and other control gears, devices and sensors, which may have more strict timing requirements in transmission of the control signals. 
Some of the key features and advantages specific to this research work, compared to other related work, examined in detail in the section that follows, are the following:

- The hardware implementation approach was designed to be easily set up, based on ARM Raspberry Pi development board, for fast construction and experimental testing purposes.

- The DALI control software is based on dynamic loadable kernel modules that provide flexibility to the overall DALI driver control system, and save RAM storage space, usually limited in embedded IoT devices.

- The control system is enhanced with hard real-time capabilities, based on PREEMPT_RT real-time patch, therefore is capable to support future deterministic timing requirements in control of electronic ballasts and other control gears.

- The Raspberry Pi3 microcontroller is easily reprogrammable and reconfigurable, supports various programming environments and therefore, DALI lighting control applications making use of the open-source developed DALI kernel module could be written in various programming languages, particularly in educational and research environments with limited budgets or technical skills, and limited resources.

This paper is structured as follows: Section 2 describes previous related work; Section 3 describes the materials, design methodology and goals, of the proposed design architecture; Section 4 presents the implementation of the proposed system architecture; Section 5 describes the software modules developed for DALI communication and control; Section 6 presents the experimental results and provides a brief discussion of the experimentations carried out and system's overall performance; and Section 7 draws conclusions.

\section{Related Work}

Reviewing the literature, many works deal with smart lighting control [28-30]. However, most of these works are based on powerful PCs and servers. For example, the work of Wang et al. [31] presents a system for a digitally-controlled ballast for ultraviolet lamps based on a ZigBee DALI bridge module that connects to a PC. Such systems, although powerful, do not have the flexibility and variability of applications provided by embedded microcontroller-based control architectures like the system proposed in this research. In addition, power requirements and cost is quite high.

Embedded systems based on microcontrollers are widely used in real world commercial, educational and research applications. In particular, applications featuring ARM-based microcontroller boards (e.g., Texas Instruments boards, NXP Semiconductors boards, Beagle Board, Raspberry Pi, etc.) with Linux as an embedded operating system are quite common and offer appropriate computing performance. The embedded microcontroller technology could be used for various lighting control purposes in various fields, in building and civil engineering [32], in vision research [33,34], in agriculture [35], in smart home [36], etc. In recent years, interesting applications in lighting control and energy-saving are implemented based on Arduino micro-controller, such as the work of Yin et al. [37], based on Arduino Mega 2560. In this work, the authors present a novel energy-saving control strategy applied to minimization of the light-energy consumption, by separately manipulating the brightness of multiple lighting sources, and realizing the desired lighting level. Arduino is not as powerful as Raspberry $\mathrm{Pi}$, but is very suitable for such control application purposes. However, the developed lighting control system does not implement the DALI communication protocol.

In our previous research work [38], we have investigated lighting control issues (e.g., dimming) in educational laboratories based on a BeagleBone Black microcontroller. In current research, the proposed system is implemented in a more versatile, cheaper and powerful microcontroller, the Raspberry Pi3, enhanced with real-time capabilities, and a Python-based graphical user interface for user friendly application control.

Lighting control systems, sensing and actuation devices, which are automatically controlled from both embedded computing devices (e.g., Raspberry Pi) and remote control units such as smart phones 
have become quite common [39-41]. The work of Leccese et al. [42] presents such a control system for full control of street lighting, based on a Raspberry Pi and a ZigBee module. This is an interesting research work, however the authors do not implement the DALI protocol in the control of the street lighting. The same applies for the work of Bannamas et al. [43], where the authors present a lighting energy management system based on Raspberry Pi, but with no DALI protocol implementation.

DALI-based lighting control systems are commonly preferred due to their advantages on performing digital lighting control operations and other management tasks (e.g., energy monitoring) [44,45]. However, most of these works are based on microprocessors other than ARM or Raspberry Pi based microcontrollers [46-49]. For example, the work of Liang et al. [50] presents a LED DALI dimming control system based on PIC 16F684 micro-controller. This micro-controller is suitable for this specific application, however it falls quite below the requirements for future experimental research, and the capabilities of the 64-bit ARMv8 multi-core CPU-based Raspberry Pi micro-controller, utilised in the system proposed in this current research. The work of Bellido-Outeiriño et al. [51] presents a lighting control system based on TI CC2530OEM control board (CPU 8051) powered from mains (230 VAC) for managing public lighting networks. The control board, although efficient, is not easily reconfigurable, neither versatile in use, having quite a high implementation cost, and a power requirement from mains power supply, which makes it restrictive for remote installations and applications. Another work of Archana et al. [52] presents a spot luminaire control unit based on a STM32F302 MCU. The implementation is focused and limited to the control of a specific motorized and movable LED luminaire, while the DALI control software is implemented in C as a user-space application, not favorable for limited in memory space embedded applications.

ARM-based board computers such as RPI3 provide more computing power, and Linux as an embedded operating system, at a low price. In general, lighting control systems based on Raspberry Pi microcontrollers, and particularly applied in LED control, are numerous [53-59], but none of them implements the DALI protocol. There is still very limited research work that implements the DALI protocol based on Raspberry $\mathrm{Pi}$, and that is dedicated to the control of DALI ballasts, for research or commercial purposes. Recently, such a work of Sinha et al. [60] investigated the communication mechanism of a DALI network of a group of lights with a Raspberry Pi over MQTT (Message Queuing Telemetry Transport) protocol, used for energy usage optimization purposes.

Compared to existing work, this paper focuses on the development of an embedded DALI lighting control system e.g., for dimming purposes, based on a Raspberry Pi3 microcontroller with real-time capabilities due to PREEMPT_RT patch, and dynamically loadable kernel modules as the DALI control software.

\section{Materials and Methods}

\subsection{The DALI Protocol}

The standardization of the signals for the controllable electronic ballasts is necessary for obtaining interchangeability between controllable ballasts. The Digitally Addressable Lighting Interface (DALI) has emerged as a standard to address growing power issues, mostly for commercial and industrial purposes. The DALI standard was defined in the IEC 60929 standard (described in Annex E) for fluorescent lamp ballasts (part 201), published by the International Electrotechnical Commission. Subsequently, it was updated to the new standard IEC 62386, to include other electronic lighting equipment, such as LED modules (part 207, IEC 62386-207:2018) [61]. DALI-2 is the latest version of the DALI protocol which includes control devices (part 103). All new parts of IEC 62386 are aligned with DALI-2.

DALI consists of a single set of control wires that form a low-voltage two-wire serial bus. This pair of wires form the bus for communication to all devices on a single DALI network. A DALI network consists of a controller and one or more DALI lighting devices (up to 64 devices with each ballast having its own unique stored address). 
The DALI controller sends 16-bit Manchester encoded (bi-phase) data packets, and the ballast can respond with an 8-bit Manchester encoded data packet. Data is transferred between controller and devices by means of an asynchronous, half-duplex, serial protocol over a two-wire differential bus, with a fixed data transfer rate of $1200 \mathrm{bit} / \mathrm{s}$. A DALI control interface voltage between $9.5 \mathrm{~V}$ and $22.5 \mathrm{~V}$ is considered a physical high signal (typical $16 \mathrm{~V}$ ), whereas a voltage in the $\pm 6.5 \mathrm{~V}$ interval (typical $0 \mathrm{~V}$ ) is taken as a physical low signal. In general, the interface voltage is high if there is no communication (idle state). The current allowed to run through a network is limited to $250 \mathrm{~mA}$ with a maximum consumption of $2 \mathrm{~mA}$ per ballast.

DALI protocol is based upon the master-slave principle. The master (DALI controller) sends messages (forward frames) to any slave device (DALI ballast) in the system, while any slave may respond with messages (backward frames) with maintenance and energy information such as the status of the ballast and luminaries.

As shown in Figure 1, a DALI message structure is made up of an address and a command. The address corresponds to one of the ballasts in the loop, and the command tells that particular ballast what to do. All modules execute commands with broadcast addresses. A frame sent by the master, called a forward frame, consists of 19 bits (a start bit, address byte, command byte and two stop bits), while a backward frame sent by the slave is an 11 bits frame (a start bit, data byte and two stop bits).

\begin{tabular}{|c|c|c|c|c|c|c|c|c|c|c|c|c|c|c|c|c|c|c|c|}
\hline \multirow{2}{*}{$\begin{array}{l}\mathrm{s}=\text { Start bit which is a logical } 1 \\
\text { YAAA AAAS = Address byte } \\
\text { XXXX XXXX = Data byte } \\
\text { st = Stop bit (Idle line) }\end{array}$} & \multicolumn{10}{|c|}{8 address bits } & \multicolumn{6}{|c|}{8 data bits } & \multicolumn{3}{|c|}{ LSB: idle } \\
\hline & idle & & Y & A & A & A & A & A & A & $\mathrm{S}$ & $x$ & $X$ & $X$ & $X$ & $x$ & $x$ & $x$ & \begin{tabular}{|c|c|c|}
$X$ & st \\
bit
\end{tabular} & \begin{tabular}{|c|} 
st \\
bit
\end{tabular} \\
\hline & & & & & $\begin{array}{l}\mathrm{Y}= \\
\mathrm{Ad} \\
\mathrm{S}=\end{array}$ & $\begin{array}{l}\text { res } \\
\text { ele }\end{array}$ & $\begin{array}{l}\text { by } \\
\text { tor }\end{array}$ & & If ' 0 & $\begin{array}{l}\text { adc } \\
\text { dat }\end{array}$ & $\begin{array}{l}\text { or } \mathrm{s} \\
\text { ress } \\
\text { ist: } 1 \\
\text { by } \\
\text { by }\end{array}$ & 111 & lire & & & & evel & $\begin{array}{l}\text { Stop } \\
\text { bit }\end{array}$ & $\begin{array}{c}\text { Stop } \\
\text { bit }\end{array}$ \\
\hline
\end{tabular}

In Backward frame ' $0 x \mathrm{FF}^{\prime}$ is considered a 'Yes'. If the line stays idle, is considered a ' $\mathrm{No}^{\prime}$. Other values vary depending on the command.

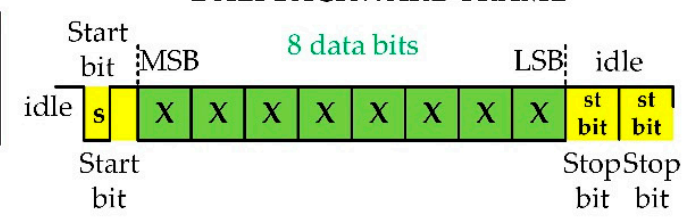

Figure 1. Digital Addressable Lighting Interface (DALI) protocol two-way communication message structure (forward \& backward frames).

DALI-based systems provide more functionality than being just typical switching and dimming devices, which justified its selection in this current research. DALI systems are very flexible and highly functional. They allow individual and grouped control of lighting, and can be scheduled when they are needed, and so provide efficient energy and cost savings. Keeping the application cost low is essential for this research work too. It is possible to extend such systems by adding other DALI controlled equipment e.g., photocells, sensors, touch screens, etc. This is quite attractive feature for future experimental research work in control of electronic ballasts and other control gears, devices and sensors. DALI lighting systems, can be easily and efficiently controlled by computing devices, as this research documents too, and provide valuable information about their status and fault conditions. Finally, DALI is an international standard, widely used in many compatible devices and ballasts produced by different manufacturers. 


\subsection{Design Overview}

\subsubsection{Essential Requirements}

A typical DALI lighting control architecture includes a microcontroller that acts as the master controller for sending lighting control instructions and receiving status information to/from the DALI ballast unit (slave) that drives the lamp/LED luminaire. The software control architecture should comply with the DALI communication protocol specifications and be capable to control most of the DALI ballast driver's operation aspects. Additionally, the system architecture must be flexible and customizable, to such extend so that to be enhanced and modified according to various user-derived system specifications and requirements.

\subsubsection{Design Goals}

The aim is to implement a low-cost and low-power open-source embedded control system for the communication and control of standard DALI ballast drivers, based on commonly available open-architecture microcontrollers such as RPi3, and being capable to adapt easily into any lighting control application needs.

A summary of the requirements the final system must fulfil are the following:

- Low-cost and low-power embedded microcontroller-based control architecture.

- Easily reprogrammable and reconfigurable according to the user lighting needs.

- Operation system with support on hard real-time functionality.

- Integration flexibility within building control panels or with other lighting control systems.

- Ability to wirelessly connect for control and monitoring purposes using standard connection protocols (e.g., Wi-Fi, Bluetooth).

- Capable to support experimental scientific research in control of electronic ballasts and other control gears, devices and sensors.

The RPi3 microcontroller presents such a low-cost sophisticated digital control device with higher level of computing capabilities among other microcontroller boards, and eventually comparable to commercial control panels. Table 1 shows some principal characteristics of commonly used low-cost but powerful microcontroller boards for research and educational purposes.

Table 1. Commonly used low-cost single board microcontrollers.

\begin{tabular}{cccc}
\hline & Raspberry Pi3 & BeagleBone Black & Arduino Uno R3 \\
\hline CPU & ARM Cortex-A53 & ARM Cortex-A8 & ATmega328P \\
(64-bit) & (32-bit) & 3-bit AVR) \\
Memory & 1 GB LPDDR2 & 512 MB DDR3 & Clash \\
Operating System & Linux & Linux & 6 analog, \\
GPIOs & 40 digital & 7 analog, & 14 digital (6 PWM) \\
& 10/100 Mbps Ethernet, & 10/100 Mbps Ethernet & via shields \\
Network & Bluetooth, Wi-Fi & 1 USB host/client, 1 USB & 1 USB, 1 UART \\
Peripherals & 4 USB hosts, & host, 4 UART & $50 \mathrm{~mA}, 3.3 \mathrm{~V}$ \\
Power Draw & 1 micro-USB power & $250 \mathrm{~mA}, 5 \mathrm{~V}$ & $35 €$ \\
Average Price & $1.34 \mathrm{~A}, 5 \mathrm{~V}$ & $49 €$ & \\
\hline
\end{tabular}

Digital ballasts control devices typically present a higher component cost largely due to power supply, or router requirements, and the need for dimmer modules, or control panels, in larger installations. The RPi3 is being extensively used for embedded applications. Although primarily is designed to function as a general processing computer, it shares many characteristics of an embedded system [62]. 
In the proposed design architecture, the RPi3 will act as the master digital lighting controller of the DALI devices in the network, capable to provide several control functions, including ballast address, ballast control, LED/ballast status, etc. It will facilitate the DALI control bus two-way communication protocol, having higher degree of communication and automation flexibility. The RPi3 microcontroller can be easily reprogrammed and reconfigured according to the user lighting needs, or for integration with other lighting control systems. The overall system could be used for a variety of purposes from private lighting control needs management (e.g., dimming control) and experimentation in small premises (e.g., flats, offices), to advanced requirements in professional buildings, including lighting usage and maintenance.

\subsubsection{Concepts and Issues}

The overall system development should be based upon a microcontroller and operating system capable to support the DALI communication mechanism in real time. DALI protocol operates at $1200 \mathrm{~Hz}$ that is a low frequency within the range of microcontrollers' frequencies and communication mechanisms. Data transfer rate is 1200 bits per second with an acceptable range of $\pm 10 \%$ (timing bit tolerance). Therefore one bit is transferred approximately every $833.333 \mu \mathrm{s}$. The DALI driver power supply needs fast response time and efficient current limiting (max $250 \mathrm{~mA}$ ). Response time of the current limiter circuit is $<10 \mu \mathrm{s}$. The response times (falling and rising slopes at low and high levels respectively) of the received and transmitted data signals at the ballast DALI (digital) terminals, should be in between $10 \mu \mathrm{s}$ and $100 \mu \mathrm{s}$. Such signal levels are considered to be reasonable for reliable operation of the DALI ballast.

In the proposed system, the RPi3 microcontroller is running UBUNTU 18.04.2 LTS (kernel version 4.15.0.1041-raspi2 \#44 Ubuntu SMP PREEMPT armv7l) and supports real-time interface circuitry with peripherals (e.g., sensors and actuators). UBUNTU Linux kernel is a low-latency preemptible kernel by default, capable to satisfy soft real-time requirements of control applications. Thus, the default scheduler cannot provide fixed and predictable latency required e.g., for real-time data sampling. Due to future application work intension, PREEMPT_RT real-time patch was installed additionally, to provide OS with deterministic scheduling and hard real-time capabilities with a fully preemptible kernel. This makes the porting of a non-time-predictable OS to a real-time environment an interesting alternative. This will ensure future experimental research work in control of electronic ballasts and other control gears, devices and sensors, which have more strict timing requirements in transmission of the signals (e.g., in vision research, and fluorescence microscopy).

\subsection{System Architecture}

The design and implementation flow of the proposed system development involves the initial specification of modules and units, their analysis, the actual programming and configuration steps, and finally real-time experimentations of the applied system for performance validation purposes.

The proposed control system consists of all the essential modules for DALI ballast communication and control, based on RPi3 microcontroller board that integrates an ARMv8 Cortex-A53 quad core processor unit within a Broadcom BCM2837 SoC [63]. The microcontroller has the ability to wirelessly connect to other devices for data transfer using standard connection protocols (Wi-Fi, Bluetooth). The overall system is tested upon the control of a DALI LED driver device (12/24 V DC, $5000 \mathrm{~mA}, 120 \mathrm{~W})$. The DALI interface bus uses voltage levels to represent the high logic level between $11.5 \mathrm{~V}$ and $22.5 \mathrm{~V}$. Therefore, the control part of the DALI voltage bus needs to be isolated. This is achieved through an opto-isolation unit (DALI Click) that acts as an interface between the ballast and the microcontroller. A dimmable $12 \mathrm{~V} 4 \mathrm{~W}$ MR16 spot LED is connected to the DALI LED driver. All the system's components, except for the DALI ballast, need a low-power supply voltage of $3.3 \mathrm{~V} \mathrm{DC}$.

The networking capabilities of the RPi3 such Ethernet, Bluetooth and Wi-Fi allow wireless communication with mobile phones running for example on Android and iOS/iPhone. Therefore, a remote-control system based on such intelligent device (e.g., tablet, smart phone) could be applied 
for monitoring and control of the RPi3-based DALI control system for luminaire management and maintenance issues. A general view of the proposed system is given in Figure 2.

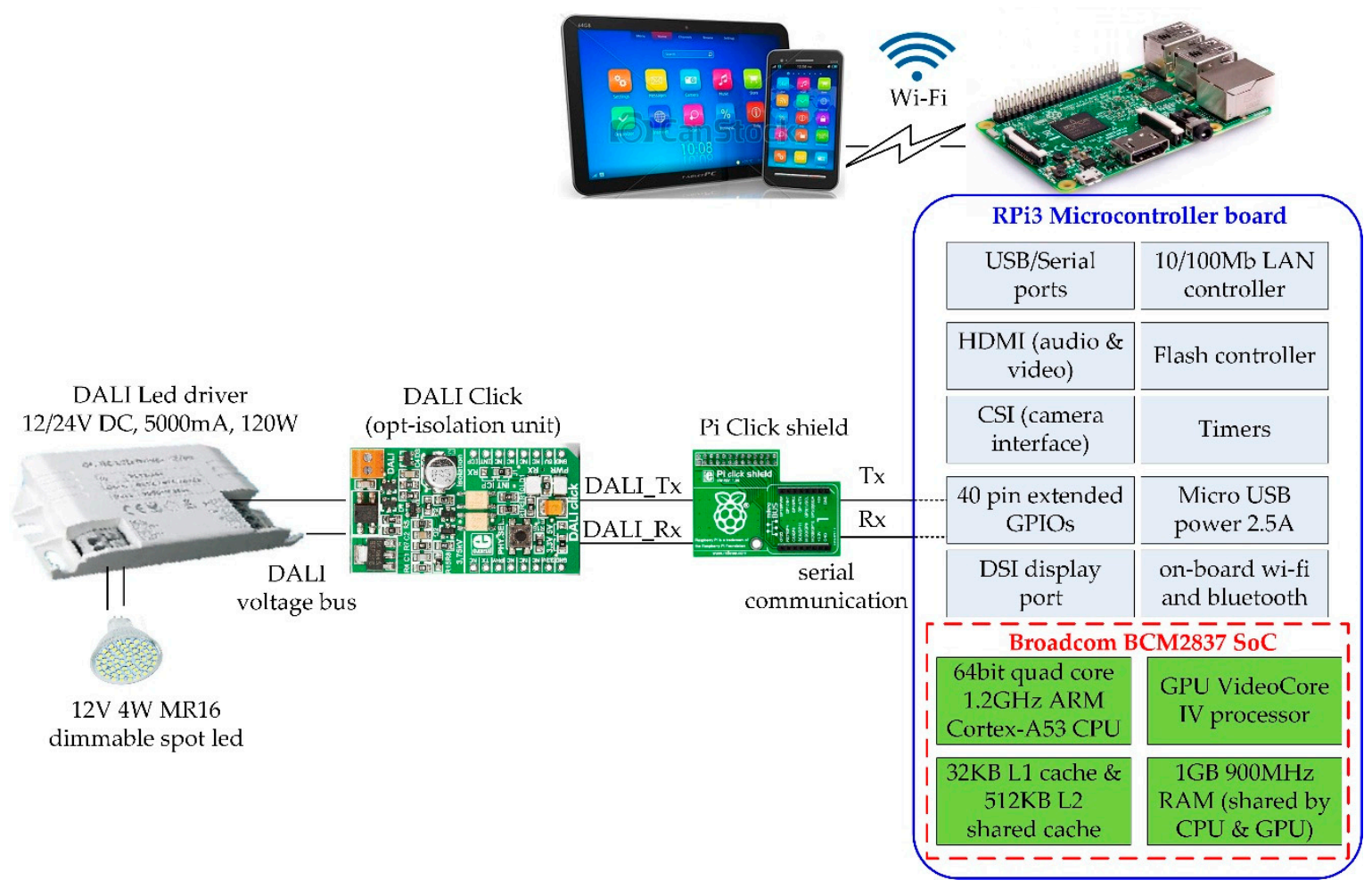

Figure 2. Raspberry Pi3 Model B (RPi3)-based DALI controller block diagram.

The kernel-based control software module developed within the microcontroller manages the DALI communication and messages conversion to/from the Manchester code. The microcontroller converts user lighting commissioning commands into acceptable formats (that conform to IEC 60929 and IEC 62386 DALI protocol standards), and routes them over a two-wire voltage interface as appropriate signals to the ballast. On physical level the communication is implemented with GPIO (General-Purpose Input/Output) interfacing. An add-on board of optocouplers provides an isolation circuit for connecting to DALI ballast that drives a dimmable spot LED.

\section{Implementation}

\subsection{Hardware}

The development of the experimental testbed platform is based on Raspberry Pi3 Model B microcontroller board, a Pi click shield, a DALI Click opto-isolation unit, a DALI LED driver and a dimmable 12 V 4 W MR16 spot LED luminaire.

\subsubsection{Microcontroller}

RPi3 is a low-cost, low-power, portable and stand-alone single board computer. RPi3 can be either powered by the USB interface or with an external power supply or a battery. RPi3 integrates a SoC based on Broadcom BCM2837, which includes an ARMv8 Cortex-A53 quad core processor at $1.2 \mathrm{GHz}$, and an 1 GB LPDDR2 $(900 \mathrm{MHz})$ memory module that facilitates data exchange between the processor and other peripheral units. Other chips on board support interface circuitry with real-time peripherals (e.g., sensors and actuators). The CPU supports ARMv8-A architecture and is capable of supporting 32-bit and 64-bit instruction sets. There are a number of GNU/Linux distributions for the Raspberry Pi, one of which is UBUNTU 18.04.2 LTS. 


\subsubsection{DALI Click Opto-Isolation Unit}

The microcontrollers do not usually have an integrated peripheral that communicates with DALI ballast drivers using the DALI protocol. Therefore, an additional opto-isolation unit is integrated to enable optically-isolated communication of the RPi3 microcontroller with the DALI ballast driver. Dali Click is a compact add-on board (by MikroElektronika) and safe solution for connecting to DALI ballasts drivers. The board communicates with the RPi microcontroller via four mikroBUS lines (RST, CS, PWM and INT) through a Pi click shield. It transfers data between the microcontroller and DALI devices over a two-wire differential bus by means of asynchronous, half-duplex, serial protocol. It features two optocouplers that provide an isolated DALI communication circuit interface. The board is powered by the RPi microcontroller with $3.3 \mathrm{~V}$ power supply.

The DALI Click adapting level circuit board is utilized as a CMOS-DALI interface with low power supply $(3.3 \mathrm{~V})$. This is because a DALI control interface voltage between $9.5 \mathrm{~V}$ and $22.5 \mathrm{~V}$ is considered a physical high signal, whereas a voltage in the $\pm 6.5 \mathrm{~V}$ interval is taken as a physical low signal. Therefore, as the microcontroller digital outputs are CMOS (0-3.3 V) it is necessary to use an adapting level circuit to convert the $3.3 \mathrm{~V}$ to the corresponding levels of the control signals.

\subsubsection{DALI LED Driver and Spot LED Luminaire}

The target ballast device used is a DALI LED driver with input voltage $12 / 24 \mathrm{~V} \mathrm{DC}$, output voltage $12 / 24 \mathrm{~V}$ DC, output current (max) $5000 \mathrm{~mA}$, LED min load $1 \mathrm{~W}$ and max power $120 \mathrm{~W}$. The electronic ballast provides the $12 \mathrm{~V} 4 \mathrm{~W}$ MR16 dimmable spot LED with a constant voltage (CV mode). LED luminaries are preferred since they have quick switching times, without the need for preheating, high lighting efficiency, and low power consumption. Table 2 shows their characteristics.

Table 2. DALI Light emitting diode (LED) driver and LED parameters.

\begin{tabular}{cccc}
\hline Parameter & DALI LED Driver & Parameter & Spot LED \\
\hline Input voltage: & DC 12-24 V & Voltage: & $12 \mathrm{~V}$ \\
Output voltage: & DC 12-24 V & Colour LED: & 2700 K warm white \\
Output current: & $5000 \mathrm{~mA}$ & Dimmable: & yes \\
Dimming frequency: & $50 \mathrm{~Hz}$ & Luminous flux: & $400 \mathrm{~lm}$ \\
Max output power: & $120 \mathrm{~W}$ & Power: & $4 \mathrm{~W}$ \\
Min load: & $1 \mathrm{~W}$ & Efficiency: & $100 \mathrm{~lm} / \mathrm{w}$ \\
\hline
\end{tabular}

\subsection{Microcontroller and DALI Click Communication}

A Pi click shield is utilized as an extension to RPi3 having a mikroBUS (an add-on board standard) host socket compatible with DALI Click boards. The mikroBUS socket comprises a pair of female headers with a pinout configuration that consists of three groups of communications pins (SPI, UART and I2C), six additional pins (PWM, Interrupt, Analog input, Reset and Cip select), and two power groups ( $3.3 \mathrm{~V}$ and $5 \mathrm{~V}$ ). Four mikroBUS lines RST, CS, PWM and INT on Pi click shield are used to establish the communication between the RPi3 and Dali Click board. RPI's GPIO14 pin out, connected to RST pin in Pi click shield, is used to transmit commands (DALI_TX) to the DALI LED driver. On the other hand, RPI's GPIO15 pin in, connected to INT pin in Pi click shield, is used to receive feedback (DALI_RX) from the DALI LED driver. Figure 3 shows the connections between DALI LED driver, Dali Click, Pi click and Raspberry Pi3 microcontroller. 


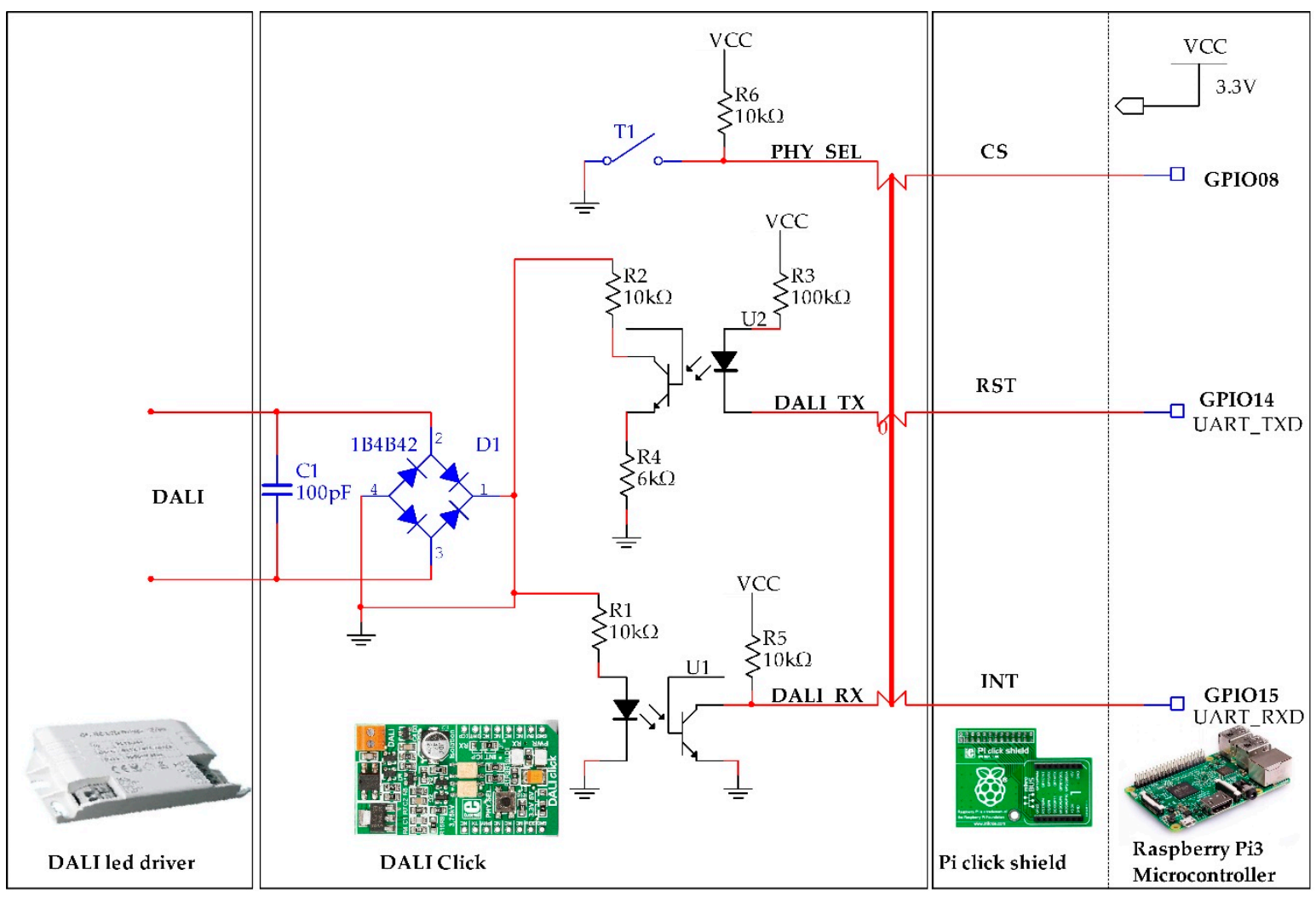

Figure 3. DALI interface and system connections.

The DALI Click board is optically isolated from the DALI LED driver and communicates with the driver via a two-wire connection. The RPi3 microcontroller communicates with Dali Click basically via two lines (DALI_TX, DALI_RX). The signals used are the transmit signal (DALI_TX) to send commands (forward frames) to the DALI ballast and the receive signal (DALI_RX) to receive ballast information (backward frames). The 3.3-V logic is translated at a higher voltage logic (16 V) on the DALI bus. This provides the appropriate voltage e.g., for dimming control purposes. The microcontroller can generate different voltage levels to control the communication and transfer the data for dimming purposes.

When a forward frame command is executed optocoupler U2 enables the transmission from the microcontroller to the DALI LED driver. The driver may react only when a query command is executed that requires a feedback. Optocoupler U1 deals with the reception of the backward frame when such a query message has been sent. A high level signal in DALI turns into low level in the microcontroller digital input DALI_RX. When DALI_TX is put in high (high level signal) diode in U2 is not biased and its phototransistor is open, giving a high level. When DALI_TX is put in low (low level signal) the LED is biased and the transistor is closed. The control software developed in the microcontroller is responsible for inverting the signals' values (due to the optocouplers' signals level inversion) and perform the Manchester encoding/decoding.

\section{DALI Communication and Control Software Modules}

\subsection{Operating System and Kernel Modules}

The embedded computer operating system is a customized version of the UBUNTU Linux operating system. UBUNTU 18.04 LTS is a Linux distribution also available for embedded IoT devices such as RPis, PDAs, Microcontrollers boards and other IoT boards. An UBUNTU 18.04.2 LTS image kernel version 4.15.0.1041-raspi2 \#44 Ubuntu SMP PREEMPT was downloaded for RPi3, and then compiled and installed (into microSD card). Some further configuration adjustments were also made to the kernel configuration in order to support kernel modules manipulation. For this purpose, kernel 
source files and make utilities were installed (apt install kernel-headers). In this way, kernel modules could be compiled and inserted into kernel memory. The build process for external loadable modules is fully integrated into the standard kernel build mechanism.

\subsection{Ballast Communication and Control Modules}

The DALI protocol communication is implemented as a kernel driver module loaded into the kernel space. Further on, user space test applications have been developed to perform the actual communication calls on module's functions to write and read from the DALI LED driver device. The DALI kernel module and applications are developed in C (using Geany as the Integrated Development Environment). A simple user interface (GUI) has been developed using the Tkinter module in Python to provide a user friendly interaction with the DALI LED driver. All the development process and compilations were executed on the microcontroller's board without any need for cross-compiling.

According to the DALI communication protocol a forward frame command consists of 19 bits (1 start bit, 1 address byte, 1 data byte and 2 stop bits). The bits are sent most significant bit (MSB) first. The packet is sent as a bi-phase Manchester encoded packet. The two stop bits have no phase change. The Manchester code is a digital encoding format in which symbol ' 1 ' is represented by a falling edge (high followed by low), and symbol ' 0 ' is represented by a rising edge (low followed by high). The backward frame sent by the slave is an 11 bits frame with the same characteristics as the forward frame ( 1 start bit, 1 data byte and 2 stop bits). DALI operation frequency is $1200 \mathrm{bps}$, which means that 1 bit time is $1 \mathrm{~s} / 1200=833,333 \mu \mathrm{s}$. Both the high and low pulses have equal width, which is equal to half the bit period $(\mathrm{Te}=416,666 \mu \mathrm{s})$.

The DALI kernel module implements the above specifications of the DALI communication protocol, that is, it facilitates the communication of the RPi3 microcontroller with the DALI LED driver. Once this module is successfully compiled and loaded into the kernel memory, afterwards any application can make use of module's function calls to communicate with and control any DALI LED device driver that comply with DALI protocol specifications.

The overall functionality of the RPi3 DALI kernel driver module is shown in Figure 4.

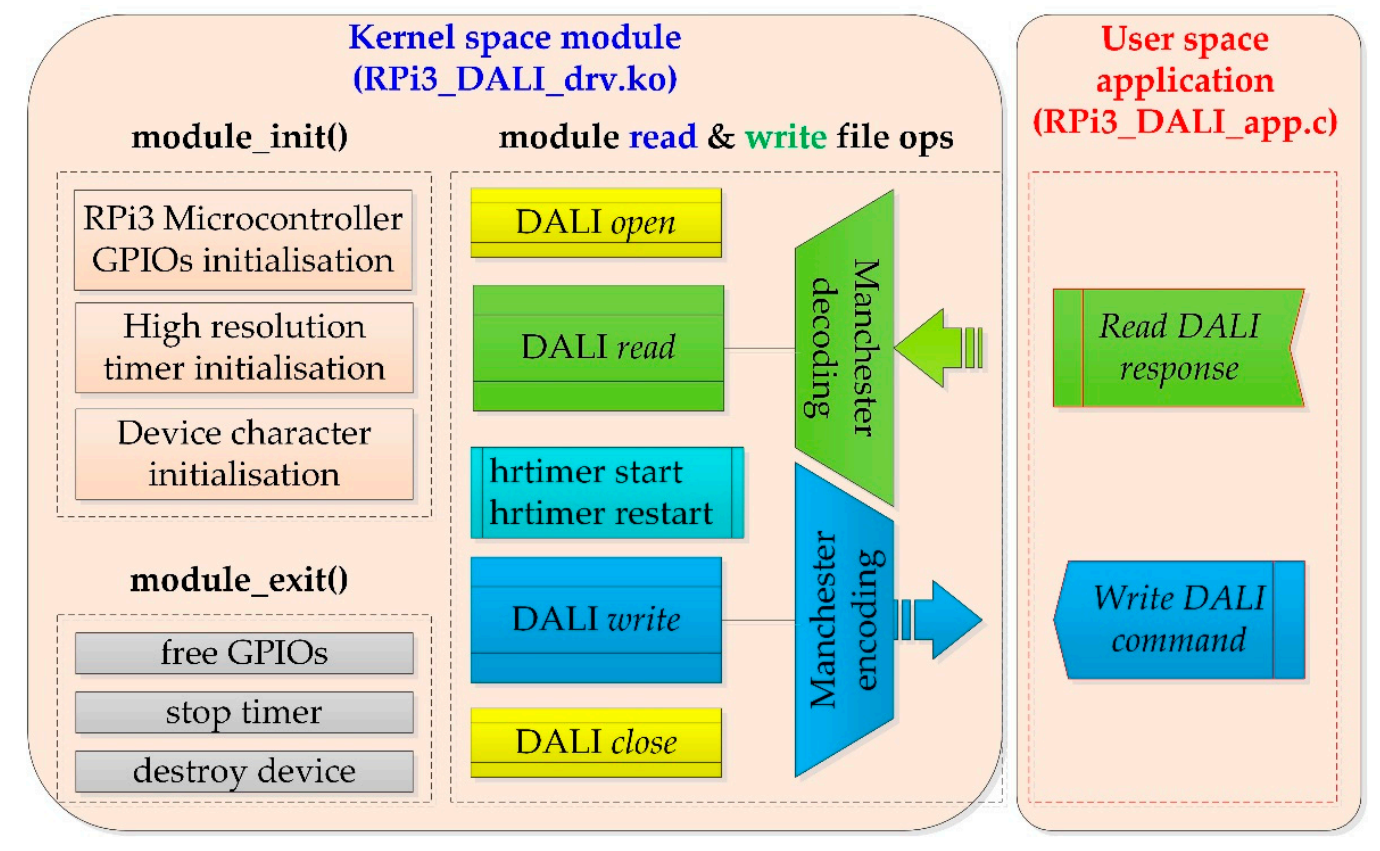

Figure 4. DALI kernel driver module functionality.

Once the module's initialization process, and verification that all components are accessible, is finished, the main tasks of this kernel module are two meaningful functions: RPi_dali_write and 
RPi_dali_read; accordingly, for sending commands to the DALI LED driver and receiving responses or information about the status of the devices. Function RPi_dali_read reads any data (ballast/LED status information) that the DALI LED driver may send back as a response to an RPi_dali_write command. Function RPi_dali_write encodes each command forward frame into Manchester coding (where two states are sent for one bit) and fills in a forward frame list that consists of two stop bits first, then a data byte, an address byte, and a start bit last. There is no phase change for the stop bits. Finally, sends the command frame with most significant bit (MSB) first through the defined as an output GPIO port to the DALI Click unit, which is connected to the DALI LED driver.

Since for every forward frame bit, two states are transmitted, and one bit transmit time is approximately $833,333 \mu \mathrm{s}$, therefore, each bit state is transmitted every $416,666 \mu \mathrm{s}$ (half bit period). A high resolution timer is initiated to facilitate this transmission at these specific time intervals. A high-resolution timer is usually a requirement in real-time systems when a task needs to occur more frequently than the $1 \mathrm{~ms}$ resolution offered with Linux. In our case, the UBUNTU kernel with PREEMPT_RT patch produces timer interrupts at exact time intervals.

The algorithm that describes the basic functionality of these DALI kernel module file operations (read and write) is shown as pseudocode in Algorithm 1:

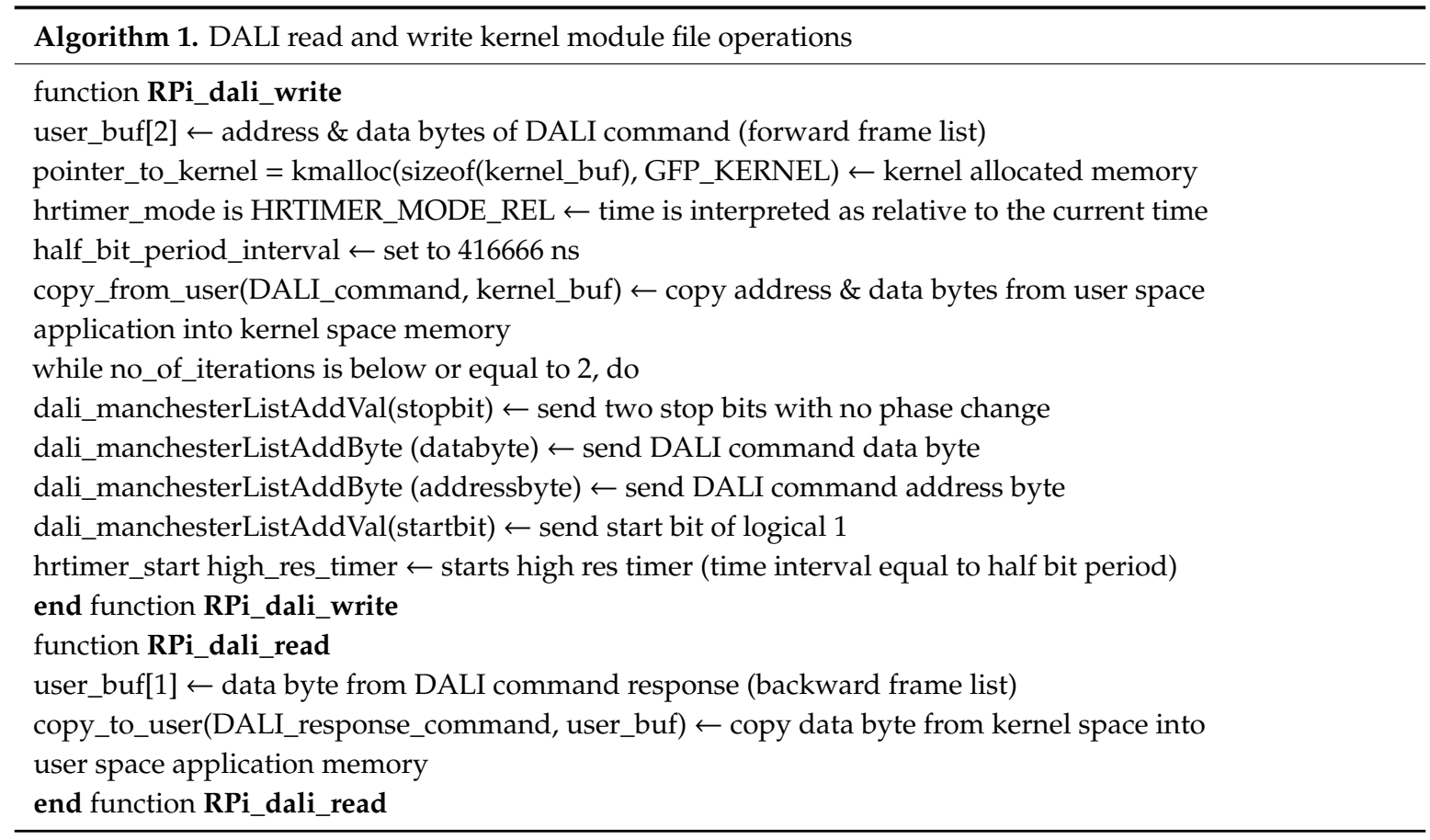

The experimental software RPi3 DALI kernel module, Python interface and C application source code developed in this research are available as an open-source project at GitHub public repository https://github.com/gadam2018/RPi3-DALI.

\section{Experimental Results}

The experiments were carried out in our Digital Systems lab capable to support the investigation of the overall system's functionality and reliability of operation. Figure $5 a, b$ shows an image of the system implemented consisting of the RPi3 board with Pi Click, the DALI Click shield, the DALI LED driver and the LED luminaire. 


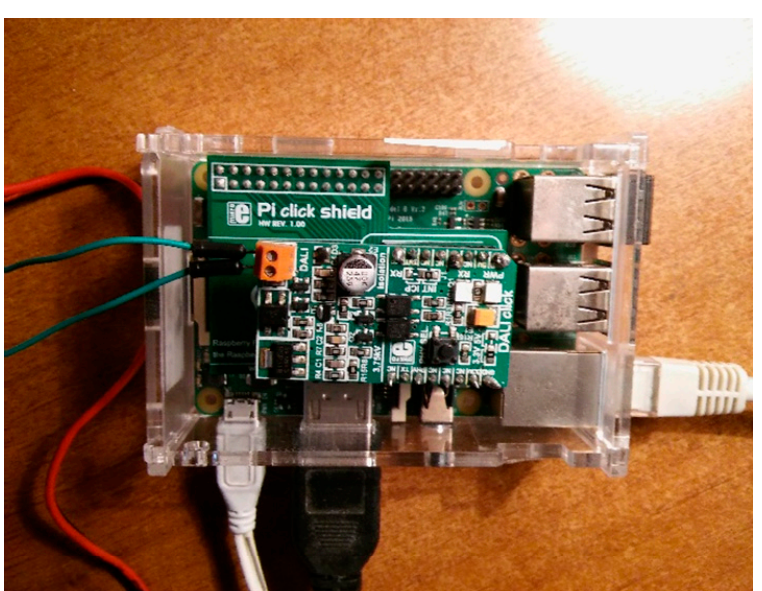

(a)

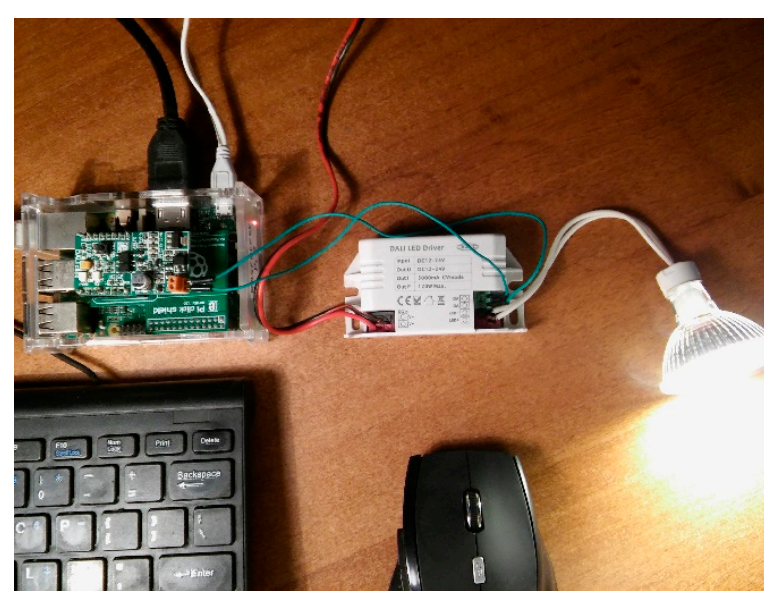

(b)

Figure 5. (a) RPi3, Pi Click and DALI Click shield; (b) the overall DALI LED Driver control system.

The system can drive any DALI LED ballast and luminaries. In particular, tests were implemented for power dimming control of LED luminaries. The experimental results provided evidence that the system performs correctly and enables appropriate communication and control through the system's DALI bus interface.

The system due to its microcontroller and operating system capabilities allows any user application to fully customize the commissioning features, such as dimming time and points, or other maintenance issues, in contrast to fixed or limited function sets provided by some commercial products.

\subsection{DALI Driver Module Application Experimental Results}

A simple graphic application (GUI), shown in Figure 6, has been developed (RPi3_DALI_Controller_interface.py) using the Tkinter module in Python to provide a user friendly interaction with the DALI ballast under control, through the C control application.

\begin{tabular}{|c|c|c|}
\hline & Address & Data \\
\hline Send command & 254 & 240 \\
\hline & & Data/Info \\
\hline Receive response & & 00 \\
\hline
\end{tabular}

Figure 6. RPi3 DALI controller simple application interface.

This Python module uses ctypes foreign function library to communicate with the DALI kernel driver by calling the C DALI_write and DALI_read functions, accordingly for Send command and Receive response buttons press, and get/set the corresponding frame fields. For this purpose, these $C$ functions are defined within a shared library (libdali.so) generated as a shared object (gcc-fPIC -shared -o libdali.so DALI_C_functions.c) from a C functions file (DALI_C_functions.c). Partial code of Python application interface and shared library is shown in Appendix A.

This simple GUI interface has been used for the transmission/reception of DALI frames. The Send command transmits DALI commands into the ballast. Two bytes (defined in the address \& data text fields) properly encoded as a forward frame are sent to the ballast. The Receive response allows the user to get information (backward frame) about the execution status of power control commands (in case a response packet is expected) or query commands (answered with a 'Yes', 'No' or 8-bit information). The DALI kernel driver module is responsible for the appropriate encoding in Manchester code format and implements the DALI communication protocol. 


\subsubsection{DALI Kernel Driver Module Dynamic Loading}

The driver module (RPi_DALI_drv.c) is built by running (make) the Makefile shown in Appendix B. This kernel driver module (RPi_DALI_drv.ko) is loaded into the kernel's memory (sudo insmod). All modules loaded into the kernel are listed in system's modules file (cat/proc/modules) and the devices directory (cat/proc/devices) as a character device. In order to make the kernel module accessible by the application of any unprivileged user, the group associated with the kernel module (e.g., root) is changed into that user's group (e.g., sudo chgrp ga /dev/RPi_DALI_drv). Then the read and write access permissions are added for that group (chmod g+rw /dev/RPi_DALI_drv) so that any user application can access this module for DALI read/write operations.

\subsubsection{Experimental Application}

The software application for communication and control experiments has been developed in user space in C (built with GNU GCC (GNU Compiler Collection) compiler). A template application code (RPi_DALI_app) that demonstrates the basic RPi3 communication and control operations (DALI read and write commands) with the DALI LED driver, through this kernel driver module, is shown in Figure 7. An example application code in C, running in user space, is shown in Appendix B.

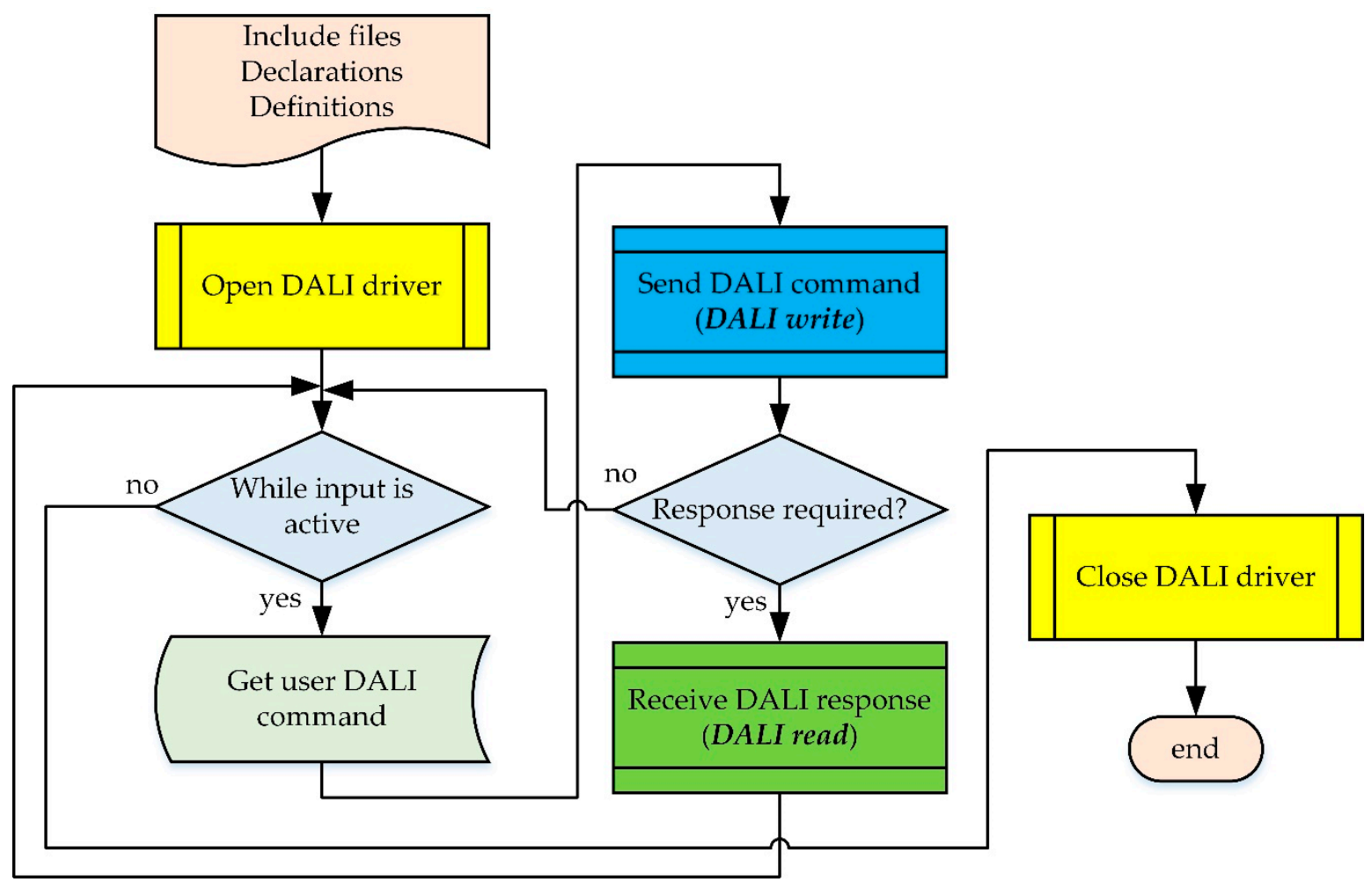

Figure 7. DALI template application program flow.

Once the DALI driver opens successfully, the main program loop (while loop) is started. The main loop runs cyclically and is used to get user input, such as DALI commands, and perform the actual transmission as forward frame commands to the DALI ballast, and receive back any response (backward frame data).

The application could be operated using the Python interface presented previously, or by using a Mate Terminal for execution, as shown in Figure 8. As an example, a dimming operation is shown, through the execution of a direct level command for setting the LED's arc power level at value of 240 (hex: FEF0, dec:254240). 


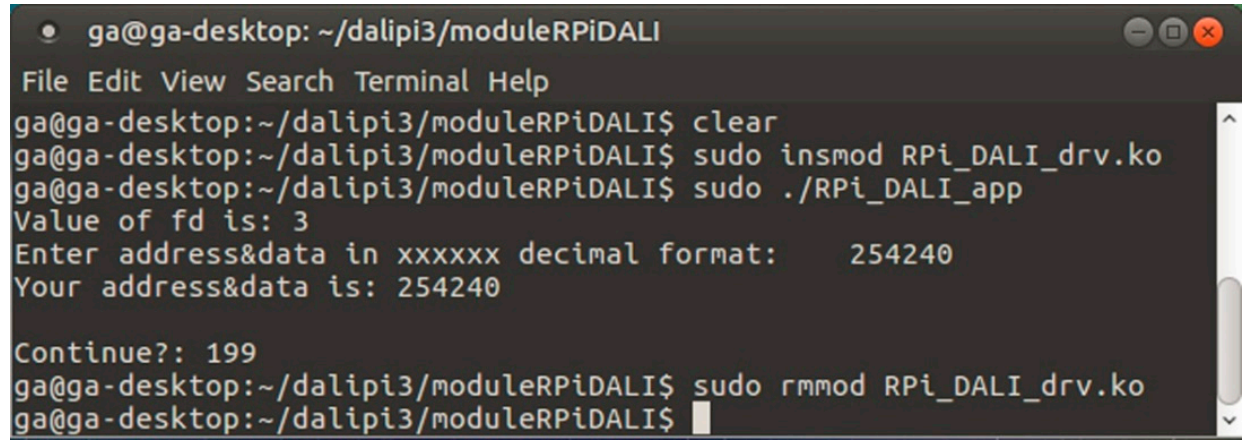

Figure 8. Console view of application execution.

Figure 9 shows a sample execution output of a direct level command for setting the LED's arc power level at value of 240 (hex: FEF0, dec:254240, bin:1111111011110000).

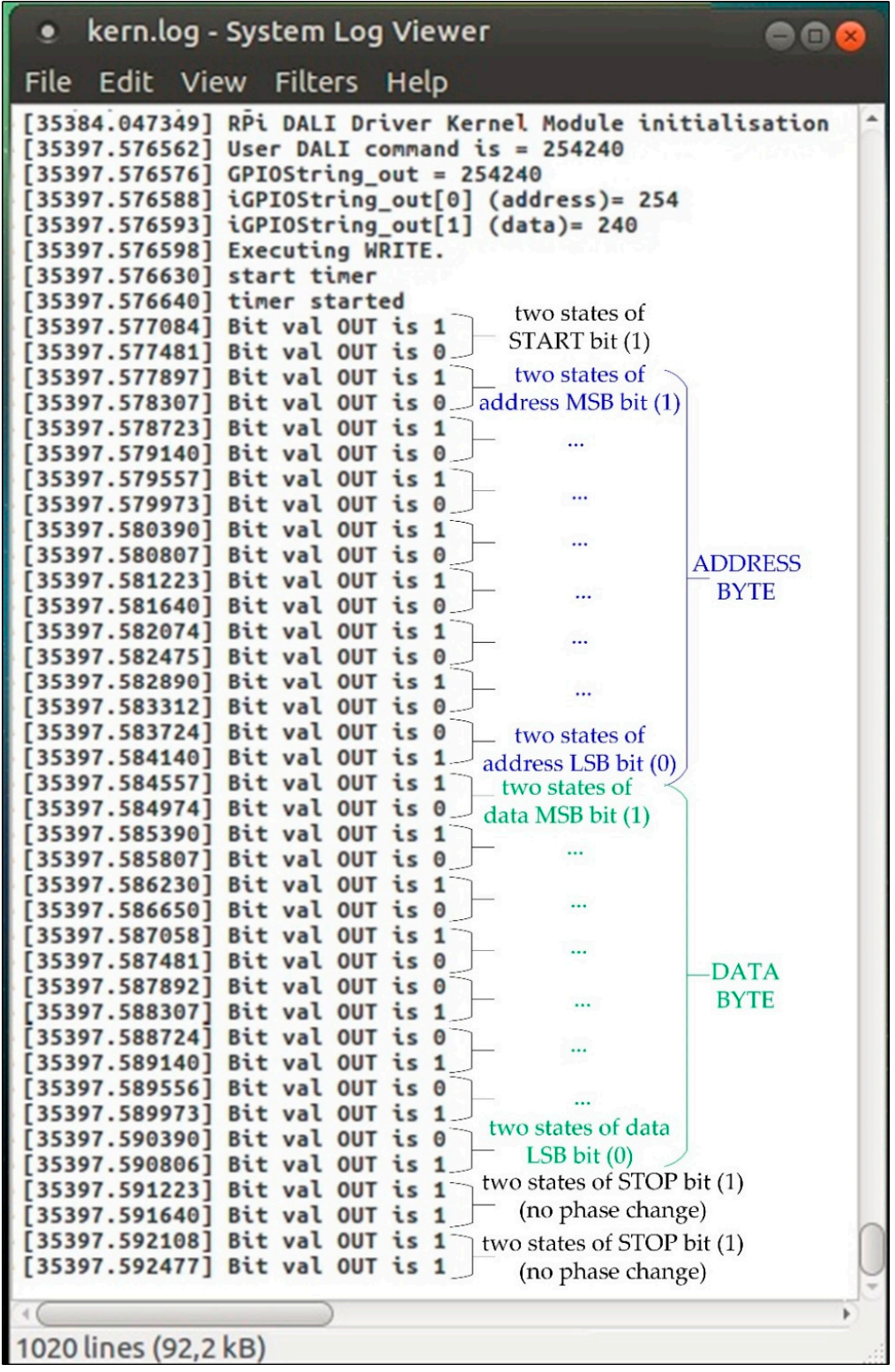

Figure 9. DALI kernel module output execution of a direct arc level command (FEF0). 
The application's format of forward_command is a decimal representation of the address and data bytes defined as a string, e.g., 254240 (hex: FEF0, dec:254240, bin:1111111011110000). The DALI driver module sends the forward_command as a DALI forward frame adding appropriate start and stop bits (with no phase change). In particular, the driver module adds into that packet of bits one start bit and two stop bits: 1111111101111000011 . Then, every bit is represented and transmitted as two states. The bits are sent MSB first. The packet is sent as a bi-phase Manchester encoded packet (two states are sent for each bit). At a terminal we can monitor the execution of any DALI command using the dmesg command or the system's Log File Viewer.

The outcome of the above experimentations proves the effectiveness of the developed kernel module for DALI communication and control of a DALI LED driver. The source code of the software applications and kernel module can be found in the Supplementary Materials.

\subsection{Discussion}

We performed the presented work in order, among other goals, to prove the concept of using a low-cost, low-power, open-source embedded control system for the communication and control of standard DALI ballast drivers, based on commonly available microcontrollers such as Raspberry Pi3. The RPi3-based DALI LED driver control system developed was shown to behave according to the design criteria and issues. The DALI control signals provided by the microcontroller were shown to operate appropriately the output of the LED under control. The whole system response of the RPi3, the DALI LED driver and the LED chip together, is in the order of microseconds, sufficient for most application purposes.

The hardware implementation approach was designed to be easily set up for fast construction and testing purposes. The design is modular, so the RPi3 DALI control module can be used to drive the majority of DALI LED drivers (DALI ballasts) allowing the end-users to select lighting devices based on their individual needs. The RPi3 supports various programming environments and therefore, DALI control applications making use of the developed DALI kernel module could be written in various programming languages.

By choosing open-source solutions both in respect to hardware (RPi3) and programming environments ( $C$, Python), the capabilities to modify the proposed RPi3-based DALI LED driver control system are greatly broadened by the active user communities that exist around Raspberry Pi (http://www.raspberrypi.org/) and Python (https://www.python.org/community/). This direction adds to the availability of the application source code as an open-source project at GitHub public repository https://github.com/gadam2018/RPi3-DALI.

The DALI LED driver control system based on the RPi3 microcontroller prototyping platform was found to be suitable for controlling LEDs in our applications. The system can be used for a wide range of DALI lighting control applications in educational and research environments with limited budgets or technical skills, and in particular for researchers, students and universities with limited resources.

\section{Conclusions}

This paper presents the implementation of a DALI LED driver control system, based on a Raspberry Pi3 Model B microcontroller board. The system runs UBUNTU 18.04.2 LTS patched with PREEMPT_RT for real-time processing. The main control software is developed as a Linux kernel module that performs all the processing, communication and control operations of the RPi3 DALI controller with the DALI ballast (DALI LED driver) and luminaire. It generates appropriately Manchester encoded control signals for digital lighting operations (e.g., dimming) and other viewing or modification purposes (e.g., maintenance tasks and energy status info). Software applications written in $\mathrm{C}$ and Python were developed for performance testing purposes.

The overall control system has the ability to operate and control DALI LED drivers and perform lighting operations (e.g., dimming). The validity of the proposed system was evaluated through actual experiments on LED driver dimming. The experimental results of the working prototype provide 
evidence that the system performs correctly. In particular, the DALI control signals by the RPi3-based DALI LED driver control system were shown to operate appropriately the output of the LED under control. The kernel modules-based control software transforms dimming commands into appropriate DALI control signals, which apply adequate dimming levels that control the illumination of the LED. For instance, in the case of these laboratory experiments, the LED's intensity is varied accordingly based on the execution of direct level commands (e.g., arc power level at value 240) setting the brightness value at different levels of the logarithmic dimming curve. The whole system response is in the order of microseconds, sufficient for most application purposes.

Some of the key features of the implemented control system can be summarized into the following:

- Open-source, low-cost, low-power, embedded control architecture, based on Raspberry Pi3, easily reprogrammed and reconfigured for integration with other lighting automation building systems.

- Linux kernel with real-time capabilities with the real-time patch PREEMPT_RT in UBUNTU, capable to support future advanced timing control requirements.

- Dynamic loadable software control modules, based on Linux kernel modules, give flexibility to the DALI driver controller and save RAM storage space, usually limited in embedded devices.

- Capable to wirelessly connect for control and monitoring purposes using one of the standard connection protocols (e.g., Wi-Fi, Bluetooth)

The system could be used for a variety of purposes from personal lighting control needs and experimental research in control of electronic ballasts and other control gears, devices and sensors, to advanced requirements in professional buildings, including energy management, lighting maintenance and usage.

Supplementary Materials: The following are available online at http:/www.mdpi.com/2079-9292/8/9/1021/s1.

Funding: This research received no external funding.

Acknowledgments: The author wants to acknowledge all the laboratory personnel and technicians for their technical support.

Conflicts of Interest: The author declares no conflict of interest.

\section{Appendix A}

/* Python application interface and shared library DALI_C_functions partial code * \#RPi3 DALI Controller Python Application Interface

import tkinter as tk

from tkinter import *

from tkinter import ttk

import ctypes

_C_DALI_func $=$ ctypes.CDLL('/home/ga/dalipi3/moduleRPiDALI/libdali.so')

class Application(tk.Frame):

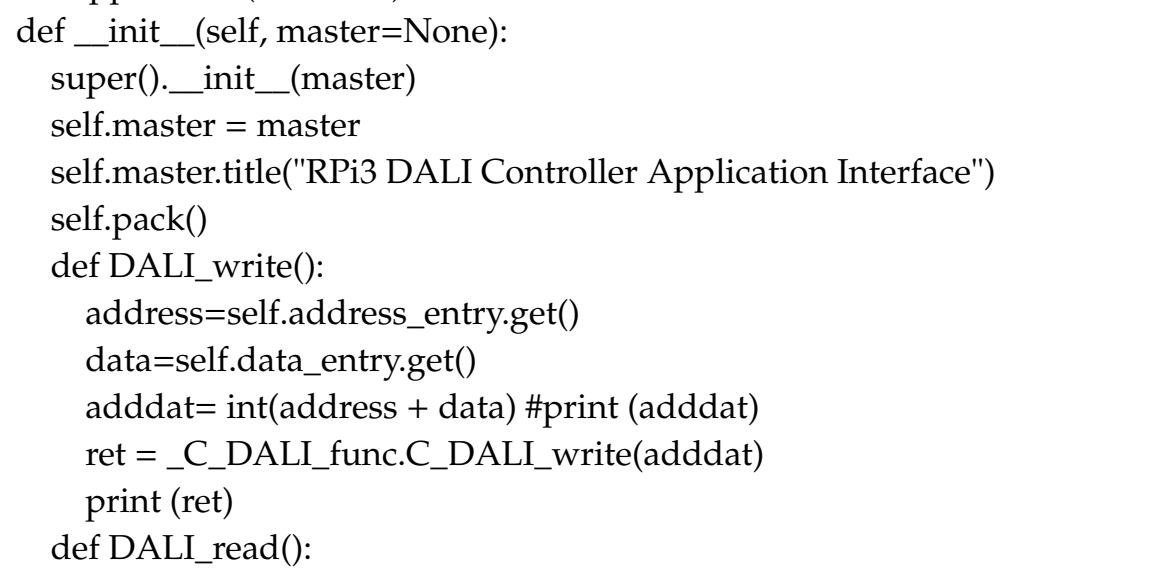




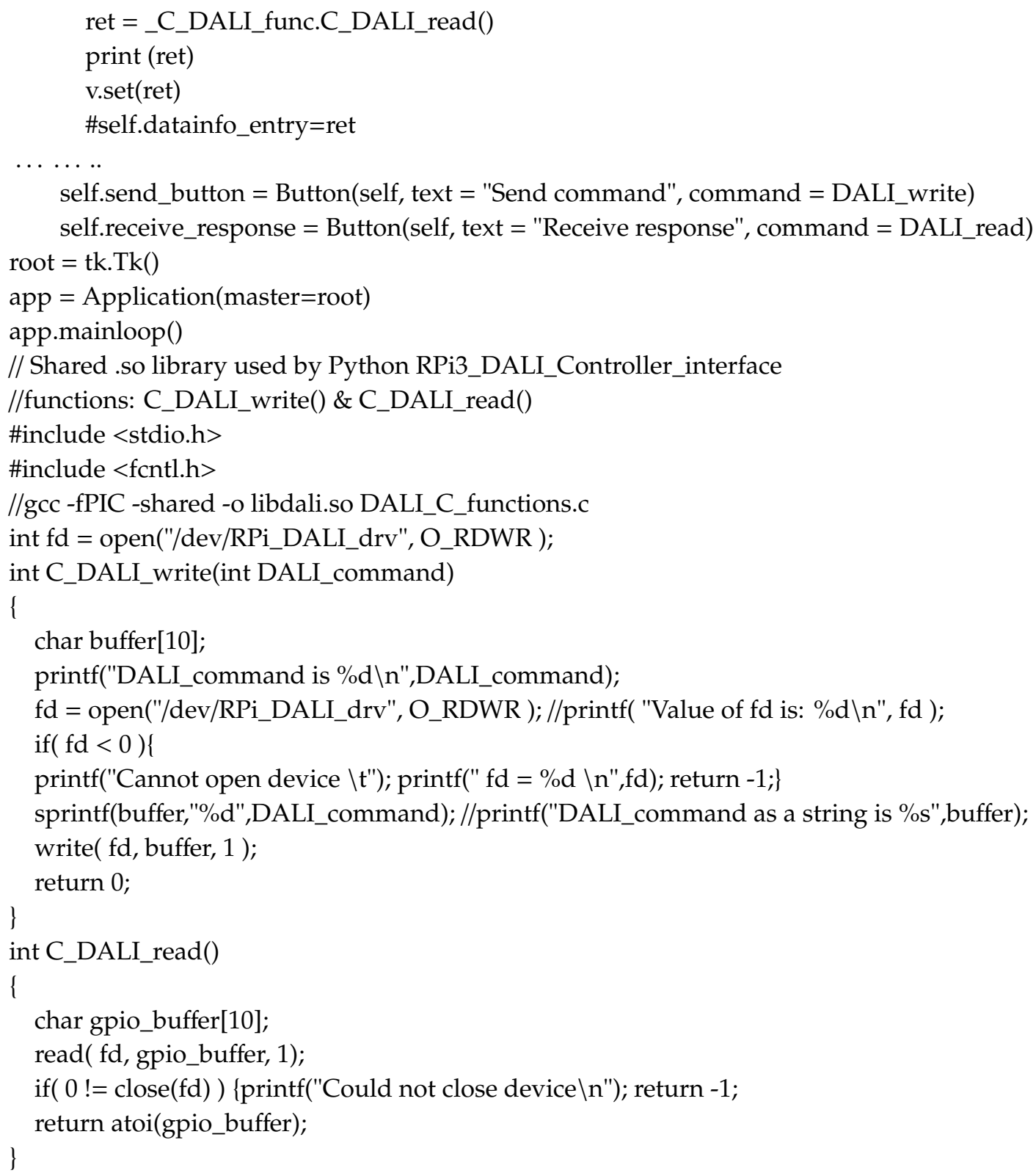

\section{Appendix B}

/* Makefile and C Application partial code*

The driver module (RPi_DALI_drv.c) was compiled (make) with the following Makefile:

KERN_SRC:=/lib/modules/\$(shell uname $-\mathrm{r}$ )/build

PWD $\quad:=\$($ shell pwd)

obj-m := RPi_DALI_drv.o

all: $\quad$ make $-C$ (KERN_SRC) $M=\$(P W D)$ modules

clean: $\quad$ make $-C$ (KERN_SRC) $M=\$(P W D)$ clean

//C example application code

\#include $<$ stdio.h $>$

\#include $<$ fentl.h $>$

\#define DALI_drv "/dev/RPi_DALI_drv" //kernel-module C name (in devices dir)

int main(void)\{

int fd = open(DALI_drv, O_RDWR );

char forward_command [10], backward_command [10]; 
while (1) \{ // decimal format of command input: Address and Data bytes as a string scanf( "\%s", forward_command);// command input, e.g., 255000, (FF 00) an indirect OFF command write( fd, forward_command, 1 ); // calls DALI_write file operation to send the command to the DALI LED driver read( fd, backward_command, 1); \}// calls DALI_read file operation to receive any answer from the DALI LED driver close $(\mathrm{fd})$; return 0 ;

\section{References}

1. Liang, Z.; Shen, H.-G. Survey on Building Energy Consumption Using Statistics Principle. In Proceedings of the International Conference on Digital Manufacturing \& Automation, Changsha, China, 18-20 December 2010; pp. 585-589. [CrossRef]

2. Yun, G.Y.; Kong, H.J.; Kim, H.; Kim, J.T. A field survey of visual comfort and lighting energy consumption in open plan offices. Energy Build. 2012, 46, 146-151. [CrossRef]

3. Gonzalo, F; Hernandez, J.A.; Moreno, B. Electrical energy consumption in buildings by means of experimental measurements: Analysis of an educational facility. In Proceedings of the International Conference on Electrical and Computing Technologies and Applications (ICECTA 2017), Ras Al Khaimah, United Arab Emirates, 21-23 November 2017. [CrossRef]

4. Bakker, C.; Aries, M.; Kort, H.; Rosemann, A. Occupancy-based lighting control in open-plan office spaces: A state-of-the-art review. Build. Environ. 2017, 112, 308-321. [CrossRef]

5. Li, D.H.; Cheung, K.; Wong, S.; Lam, T.N. An analysis of energy-efficient light fittings and lighting controls. Appl. Energy 2010, 87, 558-567. [CrossRef]

6. Soori, P.K.; Vishwas, M. Lighting control strategy for energy efficient office lighting system design. Energy Build. 2013, 66, 329-337. [CrossRef]

7. Xu, L.; Pan, Y.; Yao, Y.; Cai, D.; Huang, Z.; Linder, N. Lighting energy efficiency in offices under different control strategies. Energy Build. 2017, 138, 127-139. [CrossRef]

8. Van Driel, W.; Schuld, M.; Jacobs, B.; Commissaris, F.; Van Der Eyden, J.; Hamon, B. Lumen maintenance predictions for LED packages. Microelectron. Reliab. 2016, 62, 39-44. [CrossRef]

9. Nardelli, A.; Deuschle, E.; De Azevedo, L.D.; Pessoa, J.L.N.; Ghisi, E. Assessment of Light Emitting Diodes technology for general lighting: A critical review. Renewable Sustainable Energy Rev. 2017, 75, 368-379. [CrossRef]

10. Lee, C.K.; Li, S.; Hui, S.Y. A Design Methodology for Smart LED Lighting Systems Powered By Weakly Regulated Renewable Power Grids. IEEE Trans. Smart Grid 2011, 2, 548-554. [CrossRef]

11. Cheng, Y.-S.; Chen, J.-H.; Liu, Y.-H.; Wang, S.-C. Development of wireless RGB LED dimming control technology using smart phone. In Proceedings of the 2014 International Conference on Intelligent Green Building and Smart Grid (IGBSG 2014), Taipei, Taiwan, 23-25 April 2014. [CrossRef]

12. Su, Z. Design of White Light LED Lighting Control System. In Proceedings of the International Conference on Intelligent Transportation, Big Data \& Smart City (ICITBS 2018), Xiamen, China, 25-26 January 2018. [CrossRef]

13. Svilainis, L. LED PWM dimming linearity investigation. Displays 2008, 29, 243-249. [CrossRef]

14. Patel, M.; Mukherjee, S. Lighting Control Protocols and Standards. In Handbook of Advanced Lighting Technology; Karlicek, R., Sun, C.-C., Eds.; Springer International Publishing AG: Cham, Switzerland, 2017; pp. 559-582.

15. International Electrotechnical Commission. IEC 60929:2011. AC and/or DC-supplied electronic control gear for tubular fluorescent lamps - Performance requirements. Available online: https://webstore.iec.ch/ publication/3926 (accessed on 10 February 2018).

16. International Electrotechnical Commission. IEC 62386-201:2015. Digital Addressable Lighting Interface Part 201: Particular requirements for control gear - Fluorescent lamps. Available online: https://webstore.iec. ch/publication/22534 (accessed on 27 April 2018).

17. Ibrahim, D. Using LEDs, LCDs and GLCDs in Microcontroller Projects, 1st ed.; John Wiley \& Sons, LLC: Chichester, West Sussex, UK, 2012; ISBN 978-11-1994-070-8. 
18. Dean, A.G. Embedded Systems Fundamentals with ARM Cortex-M based Microcontrollers: A Practical Approach; ARM Education Media UK: Cambridge, UK, 2017; ISBN 978-19-1153-103-6.

19. Bai, Y. Practical Microcontroller Engineering with ARM $\neg$ Technology, 1st ed.; John Wiley \& Sons, Inc.: Hoboken, NJ, USA, 2015; ISBN 978-11-1905-237-1.

20. Jimenez, R. Microcontroller Engineer's Notebook. 12 Experiments with PIC12F683, 1st ed.; World Class Micro Electronics: Calexico, CA, USA, 2016; ISBN 978-06-9276-419-0.

21. Ferreira, J.C.; Afonso, J.A.; Monteiro, V.; Afonso, J.L. An Energy Management Platform for Public Buildings. Electronics 2018, 7, 294. [CrossRef]

22. Abdalaal, R.M.; Ho, C.N.M.; Leung, C.K.; Ohin, N.I.; Rehman, S.H. A Remotely Control Dimming System for LED Lamps with Power Factor Correction. In Proceedings of the IEEE Energy Conversion Congress and Exposition (ECCE 2018), Portland, OR, USA, 23-27 September 2018; pp. 4721-4727. [CrossRef]

23. Basil, E.; Sawant, S.D. IoT based traffic light control system using Raspberry Pi. In Proceedings of the International Conference on Energy, Communication, Data Analytics and Soft Computing (ICECDS 2017), Chennai, India, 1-2 August 2017; pp. 1078-1081. [CrossRef]

24. Urgiles, M.V.; Arpi, P.E.; Chacon-Troya, D.P. Lighting control actuator design and development for a ZigBee network with a Web server mounted on Raspberry Pi. In Proceedings of the IEEE International Conference on Automation Science and Engineering (CASE 2015), Gothenburg, Sweden, 24-28 August 2015; pp. 714-719. [CrossRef]

25. Ardito, L.; Torchiano, M. Creating and evaluating a software power model for linux single board computers. In Proceedings of the 6th International Workshop on Green and Sustainable Software, Gothenburg, Sweden, 27 May 2018; pp. 1-8. [CrossRef]

26. Membrey, P.; Veitch, D.; Chang, R.K.C. Time to Measure the Pi. In Proceedings of the ACM Internet Measurement Conference (IMC 2016), Santa Monica, CA, USA, 14-16 November 2016; pp. 327-334. [CrossRef]

27. The Linux Foundation. Real Time Linux. Available online: https://www.linuxfoundation.org/ (accessed on 24 March 2019).

28. Han, D.-M.; Lim, J.-H. Design and implementation of smart home energy management systems based on zigbee. IEEE Trans. Consum. Electron. 2010, 56, 1417-1425. [CrossRef]

29. Smith, C.B.; Parmenter, K.E. Energy Management Principles - Applications, Benefits, Savings, 2nd ed.; Elsevier: Amsterdam, Netherlands, 2015; ISBN 978-0-12-802506-2.

30. Gao, Y.; Cheng, Y.; Zhang, H.; Zou, N. Dynamic illuminance measurement and control used for smart lighting with LED. Measurement. 2019, 139, 380-386. [CrossRef]

31. Wang, S.; Liu, Y.; Chen, Y.-L.; Chen, J.-Y. Development of DALI-based electronic ballast with energy saving control for ultraviolet lamps. In Proceedings of the 2010 8th IEEE International Conference on Industrial Informatics, Osaka, Japan, 13-16 July 2010; pp. 214-219. [CrossRef]

32. Pinto, M.F.; Soares, G.M.; Mendonça, T.R.F.; Almeida, P.S.; Braga, H.A.C. Smart modules for lighting system applications and power quality measurements. In Proceedings of the 11th IEEE/IAS International Conference on Industry Applications, Juiz de Fora, Brazil, 7-10 December 2014; pp. 1-8. [CrossRef]

33. Teikari, P.; Najjar, R.P.; Malkki, H.; Knoblauch, K.; Dumortier, D.; Gronfier, C.; Cooper, H.M. An inexpensive Arduino-based LED stimulator system for vision research. J. Neurosci. Methods 2012, 211, 227-236. [CrossRef] [PubMed]

34. Nodado, J.T.G.; Morales, H.C.P.; Abugan, M.A.P.; Olisea, J.L.; Aralar, A.C.; Loresco, P.J.M. Intelligent Traffic Light System Using Computer Vision with Android Monitoring and Control. In Proceedings of the TENCON 2018-2018 IEEE Region 10 Conference, Jeju, Korea, 28-31 October 2018; pp. 2461-2466. [CrossRef]

35. Hernández-Ontiveros, J.; Inzunza-González, E.; García-Guerrero, E.; López-Bonilla, O.; Infante-Prieto, S.; Cardenas-Valdez, J.; Tlelo-Cuautle, E. Development and implementation of a fish counter by using an embedded system. Comput. Electron. Agric. 2018, 145, 53-62. [CrossRef]

36. Cheuque, C.; Baeza, F.; Marquez, G.; Calderon, J. Towards to responsive web services for smart home LED control with Raspberry Pi. A first approach. In Proceedings of the 34th International Conference of the Chilean Computer Science Society (SCCC 2015), Santiago, Chile, 9-13 November 2015; pp. 1-4. [CrossRef]

37. Yin, C.; Dadras, S.; Huang, X.; Mei, J.; Malek, H.; Cheng, Y. Energy-saving control strategy for lighting system based on multivariate extremum seeking with Newton algorithm. Energy Convers. Manage. 2017, 142, 504-522. [CrossRef] 
38. Adam, G.K.; Kontaxis, P.A.; Bouroussis, C.A.; Ventzas, D.E.; Topalis, F.V. Embedded computer communication and control of DALI LED drivers. In Proceedings of the Balkan Light Conference, Athens, Greece, 16-19 September 2015; pp. 125-130.

39. Weng, T.; Agarwal, Y. From Buildings to Smart Buildings-Sensing and Actuation to Improve Energy Efficiency. IEEE Des. Test Comput. 2012, 29, 36-44. [CrossRef]

40. Ramlee, R.A.; Othman, M.A.; Leong, M.H.; Ismail, M.M.; Ranjit, S.S.S. Smart home system using android application. In Proceedings of the International Conference of Information and Communication Technology (ICoICT 2013), Bandung, Indonesia, 20-22 March 2013; pp. 277-280. [CrossRef]

41. Khunchai, S.; Thongchaisuratkrul, C. Development of Smart Home System Controlled by Android Application. In Proceedings of the 6th International Conference on Technical Education (ICTechEd6 2019), Bangkok, Thailand, 19-20 March 2019; pp. 1-4. [CrossRef]

42. Leccese, F.; Cagnetti, M.; Trinca, D. A Smart City Application: A Fully Controlled Street Lighting Isle Based on Raspberry-Pi Card, a ZigBee Sensor Network and WiMAX. Sensors 2014, 14, 24408-24424. [CrossRef] [PubMed]

43. Bannamas, S.; Jirapong, P. An intelligent lighting energy management system for commercial and residential buildings. In Proceedings of the IEEE Innovative Smart Grid Technologies - Asia (ISGT ASIA 2015), Bangkok, Thailand, 3-16 November 2015; pp. 1-6. [CrossRef]

44. Roisin, B.; Bodart, M.; Deneyer, A.; D'Herdt, P. Lighting energy savings in offices using different control systems and their real consumption. Energy Build. 2008, 40, 514-523. [CrossRef]

45. Liu, J.; Zhang, W.; Chu, X.; Liu, Y. Fuzzy logic controller for energy savings in a smart LED lighting system considering lighting comfort and daylight. Energy Build. 2016, 127, 95-104. [CrossRef]

46. Domingo-Perez, F.; Gil-de-Castro, A.; Flores-Arias, J.M.; Bellido-Outeirino, F.J.; Moreno-Munoz, A. Lighting control system based on DALI and wireless sensor networks. In Proceedings of the IEEE PES Innovative Smart Grid Technologies (ISGT 2012), Washington, DC, USA, 16-20 January 2012; pp. 1-6. [CrossRef]

47. Madzalan, S.N.; Hamid, H.; Hussin, M.F.; Kadir, K.A. A new automatic lighting control system using DALI for unikl BMI library. In Proceedings of the International Conference on Engineering Technology and Technopreneurship (ICE2T 2017), Kuala Lumpur, Malaysia, 18-20 September 2017; pp. 1-4. [CrossRef]

48. Kudryashov, A.V.; Galishheva, E.S.; Kalinina, A.S. Lighting Control Using DALI Interface. In Proceedings of the International Conference on Industrial Engineering, Applications and Manufacturing (ICIEAM 2018), Moscow, Russia, 15-18 May 2018; pp. 1-5. [CrossRef]

49. Babu, S.; Zhou, J.; Wan, M.P.; Lamano, A.S.; Sarvaiya, J.N.; Zhang, Z.; Kumar, D.K.; Gao, C.-P.; Valliappan, S.; Goh, A.; et al. Investigation of an integrated automated blinds and dimmable lighting system for tropical climate in a rotatable testbed facility. Energy Build. 2019, 183, 356-376. [CrossRef]

50. Liang, T.J.; Huang, J.F.; Yadav, P.K. Design and implementation of dimmable LED control circuit with DALI protocol. In Proceedings of the IEEE International Conference on Power and Energy (PECon 2016), Melaka, Malaysia, 28-29 November 2016; pp. 121-126. [CrossRef]

51. Bellido-Outeiriño, F.J.; Quiles-Latorre, F.J.; Moreno-Moreno, C.D.; Flores-Arias, J.M.; Moreno-García, I.; Ortiz-López, M. Streetlight Control System Based on Wireless Communication over DALI Protocol. Sensors 2016, 16. [CrossRef] [PubMed]

52. Archana, L.; Yasin, M.; Bhagya, R. DALI based light and motor control system for movable spot luminaires. In Proceedings of the IEEE International Conference on Smart Technologies and Management for Computing, Communication, Controls, Energy and Materials (ICSTM 2017), Chennai, India, 2-4 August 2017; pp. $433-437$. [CrossRef]

53. Kurniawan, A. Raspberry Pi LED Blueprints - Design, build, and test LED-based projects using Raspberry Pi; Packt Publishing Ltd.: Birmingham, UK, 2015; ISBN 978-1-78217-575-9.

54. Khot, S.B.; Gaikwad, M.S. Development of cloud-based Light intensity monitoring system for green house using Raspberry Pi. In Proceedings of the International Conference on Computing Communication Control and automation (ICCUBEA 2016), Pune, India, 12-13 August 2016; pp. 1-4. [CrossRef]

55. Aguirre, D.; Navarrete, R.; Soto, I.; Gutierrez, S. Implementation of an emitting LED circuit in a Visible Light communications positioning system. In Proceedings of the First South American Colloquium on Visible Light Communications (SACVLC 2017), Santiago, Chile, 13 November 2017; pp. 1-4. [CrossRef]

56. Perumal, V.S.A.; Baskaran, K.; Rai, S.K. Implementation of Effective and Low-Cost Building Monitoring System(BMS) Using Raspberry Pi. Energy Procedia 2017, 143, 179-185. [CrossRef] 
57. Kandilarov, R.Y. LED Lamp with Customizable Light Regimes for Horticulture Applications. In Proceedings of the IEEE XXVII International Scientific Conference Electronics-ET, Sozopol, Bulgaria, 13-15 September 2018; pp. 1-3. [CrossRef]

58. Chen, W.; Xiong, Y.; Wang, W.; Wu, T.; Li, L.; Kang, Q.; Du, Y. Assembly of a UV-LED induced fluorescence system for rapid determination of amiloride in pharmaceutical tablet and human serum. Talanta 2019, 203, 77-82. [CrossRef] [PubMed]

59. Froiz-Míguez, I.; Fernández-Caramés, T.M.; Fraga-Lamas, P.; Castedo, L. Design, Implementation and Practical Evaluation of an IoT Home Automation System for Fog Computing Applications Based on MQTT and ZigBee-WiFi Sensor Nodes. Sensors 2018, 18, 2660. [CrossRef] [PubMed]

60. Sinha, A.; Sharma, S.; Goswami, P.; Verma, V.K.; Manas, M. Design of an energy efficient Iot enabled smart system based on DALI network over MQTT protocol. In Proceedings of the 3rd International Conference on Computational Intelligence \& Communication Technology (CICT 2017), Ghaziabad, India, 9-10 February 2017; pp. 1-5. [CrossRef]

61. International Electrotechnical Commission. IEC 62386-207:2018. Digital Addressable Lighting Interface Part 207: Particular requirements for control gear - LED modules. Available online: https://webstore.iec.ch/ publication/30618 (accessed on 12 May 2018).

62. Holt, A.; Huang, C.-Y. Embedded Operating Systems: A Practical Approach, 2nd ed.; Springer: London, UK, 2018. [CrossRef]

63. Raspberry Pi Foundation. Raspberry Pi3 Model B. Available online: https://www.raspberrypi.org/products/ raspberry-pi-3-model-b (accessed on 5 February 2017).

(C) 2019 by the author. Licensee MDPI, Basel, Switzerland. This article is an open access article distributed under the terms and conditions of the Creative Commons Attribution (CC BY) license (http://creativecommons.org/licenses/by/4.0/). 